\title{
Avian Influenza: Review
}

\author{
Bidjeh Kebkiba ${ }^{1}$; Ban-boBanbeto Antipas ${ }^{2}$; Ouagal Mahamat ${ }^{1}$ \\ I (virology and epidemiology Units, Livestock Research Institute for Development, N'Djamena, Chad) \\ ${ }^{2}$ (Department of biology, Faculty of Exact and Applied sciences, N'Djamena, Chad)
}

\begin{abstract}
Avian influenza (AI) is a contagious infectious disease caused by three RNA viruses of the family Orthomyxoviridae and genus influenza virus. There are three types of influenza viruses (A, B and C.Type A influenza virus is defined as highly pathogenic AI (HPAI) or Lowly pathogenic AI (LPAI) by its ability to cause severe disease in intravenously inoculated young chickens in the laboratory, or by its possession of certain genetic features associated with HPAI viruses.All cases of highly pathogenic avian influenza due to virus strains belong to the $\mathrm{H5}$ and H7.A (H5NI) virus isolated from animals and humans since 2003 are divided into two (2) distinct genetic groups, closely related to each other. Since the appearance of HPAI in 2003 in several South-East Asian countries, outbreaks of the disease have been recorded in Central and Eastern Europe, the Middle East and Africa. This has disastrous consequences for the poultry industry in these regions and raised serious public health concerns. Thistook global dimension, which is translating in mobilization of regional and international communities.Historically there were three pandemics of AI in the last century: The so-called Spanish flu in 1918 making between 40 and 50 million human deaths, the so-called Asian flu in 1957 causing 2 million human deaths and the so-called Hong-Kong flu in 1968 causing 1 million human deaths.The objective of the present article is to synthesize information gathered from literature. The manuscript describes nature of the disease, resistance to physical and chemical action, its epidemiology, geographical distribution episodes of antigenic shift, genetic re-reassortment process, economic and socio-economic impacts.
\end{abstract}

Key Words: avian influenza, disease, etiology, epidemiology, transmission, virus.

\section{Introduction}

The flu (or influenza) is a common and contagious infectious disease caused by three RNA viruses (A, $\mathrm{B}$ and $\mathrm{C}$ ) of the family Orthomyxoviridae and genus influenza, affecting birds and some mammals including pigs, seals and human (OIE, 2005).The word "influenza" (shortened to flu) was used for the first time in England during the epidemic of 1743 to qualify the flu. It seems to come from the Italian term "influenza di freddo" (under) the influence of cold (OIE, 2016). It recalls the seasonal nature of the disease, which suggests the influence of the external environment on man. The virus is believed to survive better outside the body in dry and cold weather, which is why seasonal epidemics occur in winter in temperate climates. Avian influenza refers to the different forms of the influenza virus that infects wild and domestic birds. The disease is also known as fowl pest. This disease is transmissible between poultry and more rarely to mammals, including pigs that are both susceptible to avian influenza and human influenza viruses, but are usually difficult to transmit to humans. Some species of birds and in particular some ducks, are often asymptomatic carriers.

Endemics have been shown to be active throughout the world, and the H5N1 avian virus seems to be adapted to temperate and cold areas (Siberia), as well as to hot zones, especially in Southeast Asia and Indonesia, with some foci in Africa, in the tropical zone.The French word flu would have a Germanic origin, Grippen meaning "grab, grasp suddenly." In other words: we do not catch the flu, it is she who "grips" us. The flu was also called "folette" in 1733.The avian flu $\mathrm{A}(\mathrm{H} 5 \mathrm{~N} 1)$ remains a serious threat. The virus, which is originally only contaminating the birds mutates very quickly. Since 1997 is known and the first human casein Hong Kong that cross the barrier of species. If the disease is not transmitted from man to man, it is fatal in $60 \%$ of cases (available on line: http://www.cfsph.iastate.edu/Factsheets/pdfs/highly_pathogenic_avian_influenzacitations.pdf).

Influenza virus infects other mammals than humans, terrestrial and marine. But it is with the bird that it is the most frequent. In animal it is long called "avian plague", "avian flu" or "chicken flu". Terminological evolution in animals is due to two reasons. The first is the identification of two groups of causal viruses of what had been called plague; hence the segmentation in Newcastle disease and influenza, the second is the decision to worry, at the sanitary level, only influenza viruses highly pathogenic to the species GallusGallus. At the symposium in Beltsville, Maryland (1981), it was decided not to speak of "chicken plague" but "highly pathogenic influenza", although the pathogenic character was not only dependent on the virus but also on immunity of the infected individual.

Avian influenza (AI) is a highly contagious viral disease affecting several species of food producing birds (chickens, turkeys, quails, guinea fowl, etc.), as well as pet birds and wild birds. Occasionally mammals, including humans, may contract avian influenza (OIE, 2003).Although avian influenza caused by highly 
pathogenic virus strains have sometimes been shown to infect man, this disease should not be confused with human influenza, a common human disease. Fowl plaque, now known to be caused highly pathogenic strains was described by Perroncito as serious disease of chickens in Italy in 1878. Centanni and Savunozzi demonstrated in 1901that the disease was caused by a filterable agent.Avian Influenza has captured the attention of the international community over the years, with outbreaks in poultry having serious consequences on both livelihoods and international trade in many countries (OIE, 2003). In addition, although most avian influenza viruses do not infect humans, some, such as avian influenza H5N1 and H7N9, are well known to the public because of their implication in serious and sometimes fatal infections in people (FAO-EMPRES, 2013).Nowadays, due to ongoing circulation of various strains (H5N1, H5N2, H5N8, H7N8, etc.), outbreaks of avian influenza continue to be a global public health concern.

It was demonstrated in 1955 that fowl plague viruses were type A influenza viruses. Virus related to the original fowl plague isolates (surface antigens-H7N1 and H7N7) caused high mortality among chickens, turkeys and other species.It has since been found that AI viruses cause a wide range of disease syndromes, ranging from severe to mild to high in domestic poultry(OIE, 2005).

There are many AI virus strains, which are usually classified into two categories according to the severity of the disease in poultry: Lowly pathogenic (LPAI) strains, which typically cause few or no clinical signs in poultryand highly pathogenic (HPAI) strains, which can cause severe clinical signs and potentially high mortality rates among poultry(OIE, 2003).Low pathogenic notifiable avian influenza (LPAI) are all viruses of H5and H7 subtypethat are not HPAI viruses. HighLly pathogenic avian inffluenza (notifiable AI viruses have an intrvenous pathogenicity index in 6-week-old chikengreater than 1.2 or, as an alternative, cause at least $75 \%$ mortality in 4to 8-week-oldchikens infected invenously. H5 and H7viruses, which do not have an IVPI greater than 1.2 or cause less $75 \%$ mortalityin an intravenous lethality test should be sequensed.This will determinewhethermultiplebasic amino acids are present at the cleavage site of the hemagglutinin molecule if the amino acidmotif is similar to that observed for other HPAI isolates, the isolate being tested should be considered as HPAI(FAO, 2009).

H5N1, for example, a highly pathogenic AI virus, was initially diagnosed in humans in Hong Kong in 1997(Capua I et al., 2002). The virus then re-emerged in 2003 and 2004, and spread from Asia to Europe and Africa causing several hundred human cases and deaths, as well as destruction of hundreds of millions of poultry (OIE, 2009). This Asiatic form of H5N1 triggered concern from scientists and authorities and remains under close surveillance due to its feared pandemic potential if a mutation allows it to be transmitted from human to human.

\section{Etiology}

Influenza viruses belong to the family Orthomyxoviridae and genusinfluenza virus. They are classified into three main types (A, B, C). Influenza type A viruses infect multiple species. Influenza type B and C both infect humans, but type $C$ is also known to infect swine. Several human influenza strains are type B while all avian strains are type A. They are considered the most virulent group, although not all strains cause clinical disease. Type AInfluenza viruses are classified into subtypes based on two surface proteins, the hemagglutinin (HA) and neuraminidase (NA). At least 16 hemagglutinins (H1 to H16), and 9 neuraminidases (N1 to N9) have been found in viruses from birds, while two additional HA and NA types have been identified, to date, only in bats (http://www.cfsph.iastate.edu/Factsheets/pdfs/highly_pathogenic avian influenza-citations.pdf).The viral $\mathrm{HA}$, and to a lesser extent the NA, are major targets for the immune response. There is ordinarily little or no cross-protection between different HA or NA types. Two important proteins present on the surface of the virus type A: Hemagglutinin (HA): Sticks the virus to cell receptors. Neuraminidase (NA): Frees the virus to infect other cells. These proteins serve as the basis for the classification of influenza viruses (e. g. Influenza A Beijing H1N1 or Panama H3N2). These proteins are always evolving.

Type Ainfluenzavirus is defined as highly pathogenic AI (HPAI) or Lowly pathogenic AI (LPAI) by its ability to cause severe disease in intravenously inoculated young chickens in the laboratory, or by its possession of certain genetic features associated with HPAI viruses. To date, the fully virulent HPAI viruses found in nature have always contained $\mathrm{H} 5$ or $\mathrm{H} 7$, although there are rare examples of other viruses that could technically be considered HPAI (OIE, 2014).Avian influenza is caused by the avian influenza virus type A and essentially two subtypes of H5 and H7 viruses among which there exist different strains more or less pathogenic. The strains differ from each other by the two types of glycoproteic spicules. Highly pathogenic strains (HPAI), only of H5 and H7 subtypes, can induce up to $100 \%$ mortality (on improved breeds of poultry) while low pathogenic strains (LPAI) are not lethal, but producing some respiratory disorders, or sometimes not causing any symptoms. 


\section{Mutations-Antigenic shift and drift in influenza A viruses}

Influenza viruses evolve and mutate according to two mechanisms: mutations (antigenic or antigen slides) or antigenic reassortment. Slip Mutations are antigenic variations that do not alter the overall antigenic structure of the virus and therefore help maintain partial immunity in the short term. These mutations occur at the time of viral RNA synthesis due to the high rate of viral RNA polymerase errors. To take intoaccount antigenic shifts, influenza vaccines are therefore prepared each year from the viral strains circulating the previous year. In February of each New Year, the World Health Organization (WHO) sets out the viral strains that will make up the next year's influenza vaccine, based on epidemiological data from surveillance of circulating influenza viruses. In 2005, WHO requested the replacement of influenza A / Fujian / 411/2003 strain (H3N2) by strain A / California / 7/2004 (H3N2) for the preparation of influenza vaccines.

Influenza viruses are RNA viruses. All subtypes exist in the avian world with viruses having highly variable pathogenicity for birds. In recent years, a highly pathogenic human virus, H5N1 (with H5 subtype hemagglutinin and N1 subtype neuraminidase) has spread as a panzootic of avian influenza and is transmitted Very rarely to man; It is then about avian flu. For the avian influenza virus, the term "H5N1" is still broad, and may include other subtypes. Indeed, currently, different viral strains circulate with very variable pathogenic powers. For example: A / chicken / Shantou / 423/2003 (H5N1) and A / bar-headed goose / Qinghai / 5/2005 (H5N1). The nomenclature should be understood as such: type / animal from which it was isolated, unless it is the man / place of isolation of the viral strain / strain number / year of isolation (subtype).Other strains (H5 or H7) are transmissible to humans without having the same pathogenicity. Other strains reach other species of mammals such as horse, pig, etc. When two different viruses infect the same animal the progeny virions can contain genomic material from each parent creating unique progeny with different infectious properties. Two influenza viruses infecting the same cell can produce 256 genetically distinct progeny; shifted virus causes pandemics and huge numbers of deaths; Shifts are re-assortments of avian and human viruses. New viruses emerge with new-to-human proteins on their surfaces (fig1).The influenza virus undergoes mutations, which mean that the virus is constantly changing. This process is called drift. Drift affects viruses that already are in worldwide circulation. The process allows influenza viruses to change and re-infectpeople repeatedly and is the reason virus strains in the vaccine must be updated each year. Much more rarely, the $\mathrm{H}$ and $\mathrm{N}$ proteins are not modified, but change altogether. When this happens it is called shift, and the result is a virus to which most people have no pre-existing antibody protection. When this happens one of the key conditions for a pandemic to happen has been met.There were four episodes of antigenic shift in the last century (AI type A). Episodes of antigenic shift in the last century and genetic re-reassortment process are shown in table 1 and figure 1 below.

Table 1. Episodes of antigenic shift in the last century

\begin{tabular}{|l|l|l|}
\hline Year & Influenza A & Comment \\
\hline 1918 & H1N1 & Spanishflu \\
\hline 1957 & H2N2 & Asian flu \\
\hline 1968 & H3N2 & Hong-kongflu \\
\hline 1977 & H1N1 & Beijing flu \\
\hline
\end{tabular}

Figure 1.Genetic Re-assortment Influenza A Virus Antigenic shift

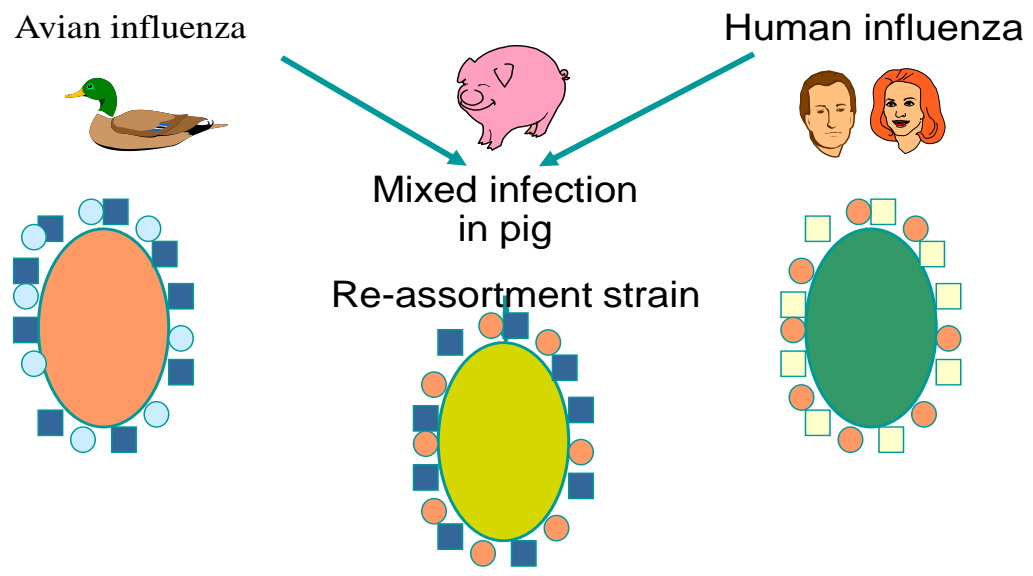




\section{Resistance to physical and chemical action}

Avian influenza viruses are relatively sensitive to inactivation by lipid solvents (detergents). Their Infectivity is rapidly destroyed by formalin, beta-propriolactone, oxidizing agents, dilute acids, ether, and sodium desoxycholate, hydroxylamine, and ammonium ions. AI viruses are inactivated at temperature of $56^{\circ} \mathrm{c} / 3$ hours and $60^{\circ} \mathrm{c} / 30 \mathrm{~min}$. Acid $\mathrm{P}^{\mathrm{H}}$ inactivates the viruses, by cons, they survived for long period in tissues, faeces and water.

\section{Natural host}

\section{Epidemiology}

All domestic and wild avian species (especially migratory birds of the family Anatidae and water birds are sensitive to avian influenza. The most sensitive domestic species are adults: Chickens, turkeys, faintly pheasants, peacocks, quails or guinea fowl. Domestic ducks appear to be resistant to AI viruses or less susceptible. Some of the viruses isolated from birds can infect horses, humans, rats, mice, mink, ferrets, pigs, cats, tigers and dogs. In aquatic birds, domestic ducks or wild ducks, infection may be with or without clinical signs.

Turkeys and Chickens are the most frequently involved in disease outbreaks. A particular strain may produce severe disease in turkeys, but not in chickens or any other avian species. Many species of wild birds, particularly waterfowl and sea birds, are also susceptible, but infections in these birds are generally subclinical. AI Viruses were isolated from domestic and wild avian species such as guinea fowl, domestic geese, quail, pheasant, parrots, gulls, shorebirds, seabirds, etc. Pigs, ferrets, cats, mink, monkeys and humans can be affected also by AI viruses. According to Capua I et al. (2000), AI (H5N1) virus occurred In Italy from 1999 to2000 in intensively reared chickens and turkeys.Migratory waterfowl particularly ducks have yielded more viruses than any other group, while domestic turkeys and chickens have experienced the most substantial diseases problems due to influenza.Highly pathogenic AI isolates have been obtained primarily from chickens and turkeys. It is reasonable to assume that all avian species are susceptible to infection.

\section{Transmission}

Initial source of infection, other poultry, migratory waterfowl, pet birds, spread by aerosol, shared drinking water, fomites, Virus in respiratory secretions and feces, Virus present in eggs but eggs unlikely to survive and hatch. In birds, avian influenza viruses are shed in the feces and respiratory secretions. Fecal-oral transmission is the predominant means of spread in aquatic wild bird reservoirs. Respiratory transmission is thought to be unimportant in most wild birds, but it can occur with a few viruses or in some hosts, particularly those that live on land. All susceptible species can eventually act as reservoirs and thus maintain nonpathogenic strains which, following mutation or recombination (mixed infection), can become pathogenic to domestic poultry. The main species are domestic palmipeds. Wild avifauna is not a reservoir of the HPAI virus. Wild Pets and exotic birds, dogs, cats, mice consuming the corpses of birds; Ticks, flies, earthworms can be contaminated by AI viruses.

Contamination of birds is mainly through direct contact with respiratory secretions and feces of sick birds (air suction loaded viral particles (excretion or droppings of dust). The contaminated feces can remain infectious for several months; indirect contact by exposure to contaminated materials (food, water, equipment, clothing, etc.). The consumption of food contaminated by excretions (droppings, streams) is possible, but not proven. Spread from one place to another is through infected animals, working materials, humans (staff and visitors), domestic and wild animals that have been in contact with contaminated poultry. Confined spaces favor the transmission of the virus, and close contacts, prolonged and repeated with infected live birds (farms, markets),Surfaces and / or objects contaminated by manure, Dried manure dust from infected animals (confined medium).

Ecological data indicate that various migratory waterfowl, sea birds, and shore birds are the reservoir for all avian influenza viruses. In addition, epidemiological and molecular genetic evidence supports the hypothesis that these aquatic birds are generally responsible for introducing the LPAI viruses into poultry. Once introduced into poultry, the viruses adapt to poultry and are spread from flock to flock or village to village by human endeavour such as the movement of infected birds. Most of outbreaks start with direct or indirect contact of domestic poultry with water birds. Direct contact between infected and susceptible birds. Indirect contact including aerosol (droplets) or exposure to virus contaminated fomites, equipment, shoes clothing, egg flats, feed trucks, and service crews. No evidence of vertical transmission, however, virus can be present within or on the surface of eggs when the hen is infected

\section{Sources of virus}

Viruses are excreted 1 to 21 days before the first signs appear on the affected birds. Sick birds are the main sources of the AI virus, which can be contaminated for more than 7 days. Domestic and wild birds that are 
not sensitive are also sources of AI viruses, such as palmipeds. The sources of virus are faeces, ocular and nasal discharge (secretions) where the virus may remain viable for long periods. Categories of sources of primary introduction for domestic poultry are other species of domestic poultry, exotic captive birds, wild birds and other animals. Highly pathogenic AI viruses may remain viable for long periods of time in infected faeces, but also in tissues and water.

\section{Incubation period}

The incubation period for an individual bird is usually 1-7 days, and up to 14 days in a flock depending upon the isolate, the dose of virus, the route of exposure, the species exposed and the age of bird. However, OIE recognizes a 21- day incubation period, which takes into account the transmission dynamics of the virus within a population. For LPAI and HPAI, the infectious period of time that virus is shed from infected birds- may be a more appropriate concept for control of the disease.

\section{Morbidity and mortality}

As variable as the signs and morbidity and mortality are dependent upon the species and virus, as well as age, environment and concurrent infections. Morbidity rates generally are poorly defined, largely because of the very large size of flocks involved and the ill-defined signs of disease in many of the outbreaks. The most virulent viruses cause HPAI (H5 and H7), in which mortality may be high as $100 \%$. Other viruses cause a much milder (primarily respiratory) disease designated "low pathogenic avian influenza (LPAI)".

\section{Clinical signs}

The clinical signs of AI are extremely variable and influenced greatly by the virulence of the viruses involved, the species infected, age, concurrent viral or bacterial disease and the environment. The infections may be asymptomatic in poultry (including domestic ducks) with detection only after serological examination. With HPAI viruses there are pronounced depression and decreased activity Decreased feed consumption, excessively watery eyes and sinusitis, cyanosis of the combs, wattle and shanks, emaciation, increased broodiness of hens and decreased egg production, oedema of head and face, diarrhoea, ruffled feathers, mild to severe respiratory signs such as coughing, sneezing, rales and excessive lacrimation, nervous disorder, cyanosis of unfeathered skin. Some birds are found dead prior to observance of any clinical signs. There may be neurological signs and reduction in normal vocalizations. Depression is common as is a precipitous drop in egg production. Respiratory signs are less prominent but can include rales, sneezing and coughing. In mature chickens, the combs and wattles are often swollen and may be cyanotic. Conjunctivitis, oedema of the head and neck, coughing, sneezing and nasal discharge may also be seen. Egg production in hens stops; the last eggs laid often have no shells. Death is common, but severely affected hens occasionally recover.

With LPAI, clinical signs in chickens and turkeys range from unapparent to mild or severe respiratory disease and can be confused with infectious laryngothracheitis and other respiratory tract infections. Mortality ranges from $3 \%$ in caged hens (layers) to $15 \%$ in meat chickens (broilers). Egg production can drop sometimes to $45 \%$ of the expected egg yield of a large flock.

\section{Pathogenicity}

The pathogenicity of the avian virus strains varies greatly. The stratification of strains is essential to distinguish low pathogenic strains from highly pathogenic strains. low pathogenic strains kill 2 to $3 \%$ of infected poultry; 2 to $3 \%$ of industrial chickens, but in case of bacterial or viral co-infections up to more than $40 \%$ In young poults (less than 35 days old) and up to $20 \%$ In breeding turkeys. Highlypathogenic strains induce the same symptoms but much more severe, possibly generalized petechiae and hemorrhages in all organs, oedema of the head and neck (visible) and lungs (less visible). If the strain is highly pathogenic, or the conditions lend themselves to infection, up to $100 \%$ (In 48 to 72 hours) of a livestock can then die. Death can also be brutal without clinical signs.More than in the turkeys, there are sometimes also in the guinea fowl neurological signs (torsion of the neck, torticollisand paralysis of limbs ...). A respiratory form of influenza corresponds to the infection of ratites.

\section{Risk factors}

The emergence of Avian Influenza depends upon Production systems (mixed farming) globalization and international trade of poultry / products (legal and illegal), Sociocultural and economic practices, including marketing in bird markets living, environment; The highly pathogenic virus can survive for a long time in the environment, especially at low temperatures, in bird droppings it can survive for at least 35 days at low temperature $\left(4^{\circ} \mathrm{C}\right)$. Wild birds may carry avian influenza viruses in their respiratory or intestinal systems without usually Present the clinical signs of the disease.Human populations at risk of contamination by the avian influenza virus are those in regular contact with the sick poultry include: Breeders and their families, 
Sellers of live poultry, Sinners, exposed to droppings in stained water environment, veterinarians and livestock technicians, personnel collecting live poultry before slaughter and carcasses (renderers), Cleaning and disinfection teams Poultry farms, Technical staff of diagnostic and research laboratories.

\section{Geographical distribution}

LPAI viruses are cosmopolitan in wild birds. Different viral lineages circulate in North America and Eurasia, although assortment occurs between these lineages at some locations. LPAI viruses are usually absent from commercial poultry in developed countries, but they may be present in other domesticated birds. The H9N2 viruses circulating in poultry are currently limited to Eurasia. The zoonotic H7N9 LPAI viruses causing outbreaks in mainland China have not been reported from other regions, except as imported cases in traveler (OIE, 2014). In 1997, a HPAI emerged in Southeast Asia and spread throughout numerous Asian, Middle, Eastern, African and European countries (OIE, 2017). According to OIE (2007), since 2003, HPAI affected 62 countries in the world out of them 12 countries in Africa. In 2015, countries likeLibya, Nigeria, Cameroon, Niger and Burkina Faso have reported to OIE cases of HPAI A(H5N1) and in 2017 Cameroon, Uganda and Egypt have reported to OIE cases of HPAI A(H5N8); Tunisia has reported case of HPAI A(H5) South Africa reported case of LPAI A(H7N2) (OIE, 2017).

AI viruses are distributed throughout the world in many domestic Birds and in wild species. Migratory waterfowl particularly ducks have yielded more viruses than any other group, while domestic turkeys and chickens have experienced the most substantial diseases problems due to influenza. Disease outbreaks with the strains H7N1 and H7N7 have been reported in many areas of the world during 20th century: North and South America, North Africa, the Middle and Far East, Europe, Great Britain, and the former Soviet Union (FAO, 2009). Highly H5 subtype was detected in chickens in Scotland and common terns. Recent serious epidemics have erupted in Italy, Hong-Kong from 1997 to 2003, Chile and Netherlands in 2003 (OIE, 2009). Following the outbreak in China and Hong Kong, 18 people were infected and 6 people died of an avian flu strain directly contracted from infected birds. Human infections and deaths caused by particular virus strain, H5N1, have been reported in Vietnam and Thailand, which has also reported the case and deaths in other non-avian species, e.g. cats.

AI first was identified over 100 years ago since then it emerged at irregular intervals in the world over recent occurrences of AI crisis. There have been 24 epidemics of HPAI since 1960, 2 in Africa (South Africa, H5N2) and current H5N1 in Hong-Kong, Korea in 1997, Japan and The Netherlands in 2003, in Asian countries. In Europe the disease occurs in 2004-2005 and onwards. HPAI (H5N1) enters in Africa in 2006 and continuing. According to FAO (2008), since 2003,10Africancountries affected more than150 million dead or killed birds. This had huge social and commercial impacts; human health dimension with 79 deaths out over 147 cases (over $50 \%$ cases of fatality rate). H5N1, for example, a highly pathogenic AI virus, was initially diagnosed in humans in Hong-Kong in 1997, the virus then re-emerged in 2003 and 2004(Chen Deng et al, 2004), and spread from Asia to Europe and Africa. In February 2006, the disease has been reported in the African continent with the first notification of the HPAI (H5N1) strain in Nigeria. From that date to 2009, 12 African countries were infected by the virus A (H5N1) with 89 human cases registered in Egypt, Nigeria and Djibouti (OIE, 2010).

\section{Direct impact}

\section{Economic Impact}

Economic losses from avian influenza vary depending on the strain of virus, species of bird infected, number of farms involved, control methods used and the speed of implementation of control or eradication strategies. Direct losses include depopulation and disposal costs, high morbidity and mortality losses, quarantine and surveillance costs and compensation paid for elimination of birds. In most developed countries, HPAI and LPAI have not been endemic diseases in the commercial poultry industries. LPAI outbreaks have caused significant economic losses. Losses from controlling seasonal outbreaks of LPAI in Minnesota between 1978 and 2003 cost growers a total of $\$ 22$ million U.S.

The 1983 outbreak of HPAI (H5N2) in the northeastern United States resulted in losses of nearly $\$ 65$ million, the destruction of more than 17 million birds, and a 30\% increase in egg prices. In the 1999-2000 outbreak of HPAI (H7N1) in Italy the government paid farmers \$100 million (U.S.) The 1997 outbreak of HPAI (H5N1) in Hong Kong live poultry market cost $\$ 13$ million (U.S.) for depopulation and compensation for 1.4 million birds. The 2001 outbreak, also in Hong Kong cost \$3.8 million dollars and 1.2 million birds were destroyed. Compensation for 18 million birds with total indirect losses at $\$ 500$ million.

\section{Indirect impact:}

The greater threat to wild birds may not be due to direct mortality from $\mathrm{H} 5 \mathrm{~N} 1$ but rather due to inappropriate government or public responses to avian influenza. Avian influenza hysteria has led to some governments and members of the public calls for nest destruction, flushing birds away from habitats as well as 
culling. Flushing or culling birds can have much greater impacts on bird populations, and could also cause infected birds to disperse and spread H5N1 virus over a larger area.

\section{Socio-Economic Impact}

Market shocks and negative consequences for livelihoods as a result of the disease and control processes applied to control it; and changes to the structure of poultry market chains, induced either by heightened biosecurity regulations created through government policy. This includes reduction of global trade in poultry (bans) by $80 \%$; Increase of poultry products by $20 \%$ socio-economic impact.

\section{Conclusion}

From 2003 to 2009, 62 countries have experienced of HPAI out of them 12 in Africa. There is evidence that $\mathrm{H} 5$ viruses of LPAI may mutate and become HPAI. There have been no HPAI outbreaks reported in Australia, New Zealand, The Pacific Community Papua and New Guinea. In Africa, Malawi, Mozambique, Zimbabwe and Chad have never reported HPAI (H5N1) outbreaks. The avian flu H5N1 remains a serious threat. The virus, which is originally only contaminating the birds mutates very quickly. Since 1997 is known and the first human casein Hong-Kong that he can cross the barrier of species. If the disease is not transmitted from man to man, it is fatal in $60 \%$ of cases.Type A and B viruses are responsible for annual influenza epidemics, but only type A viruses are responsible for influenza pandemics. Type $\mathrm{C}$ virus appears to be linked to sporadic cases and most often gives a moderate expression of influenza. Viruses A and C infect several species, whereas virus B is almost specific to the human species (it is only found in seals). Wild birds may carry avian influenza viruses in their respiratory or intestinal systems without usually Present the clinical signs of the disease.In suraigue forms, Sudden death in few hours without clinical signs is observed.

\section{References}

[1]. World Organization for Animal Health (OIE), Avian Influenza, International terrestrialCode (2016).

[2]. http://www.cfsph.iastate.edu/Factsheets/pdfs/highly_pathogenic_avian_influenza-citations.pdf.

[3]. World Organization for Animal Health (OIE), International terrestrial Code (2013).

[4]. World Organization for Animal Health (OIE), Avian Influenza, International terrestrial Code (2014).

[5]. World Organization for Animal Health (OIE), Avian Influenza, International terrestrial Code (2017).

[6]. Food and Agriculture Organization of the United Nations, FAO-AIDE News (2004). Highly pathogenic avian influenza: Overview.

[7]. Food and Agriculture Organization of the United Nations, FAO-AIDE News (2009). Highly pathogenic Avian Influenza: Situation update.

[8]. Food and Agriculture Organization of the United Nations (FAO), EMPRES- Animal Health (2013). The Avian Influenza A(H7N9) events in Eastern China, and FAO response, 360 (42).

[9]. Abolnik C, Olivier AJ, Grewar J, Gers S, Romito M. Molecularanalysis of the 2011 HPAI H5N2 (2012). Outbreak in ostriches, SouthAfrica. Avian Dis.;56(4 Suppl):865-79.

[10]. Food and Agriculture Organization of the United Nations(FAO), Empres (2009). HPAI (H5N1)-overview.

[11]. Capua I.; Alexander D.J. and al (2002). Avian influenza and human health, Acta Trop. 83, 1-6.

[12]. Chen H.; Den G. et al (2006). Properties and Dissemination of H5N1 viruses isolated during an AI outbreak in migratory waterfowl in western China. Journal of virology (80): 5976-5983. 